\title{
COMPUTING THE HILBERT TRANSFORM ON THE REAL LINE
}

\author{
J.A.C. WEIDEMAN
}

\begin{abstract}
We introduce a new method for computing the Hilbert transform on the real line. It is a collocation method, based on an expansion in rational eigenfunctions of the Hilbert transform operator, and implemented through the Fast Fourier Transform. An error analysis is given, and convergence rates for some simple classes of functions are established. Numerical tests indicate that the method compares favorably with existing methods.
\end{abstract}

\section{INTRODUCTION}

The Hilbert transform of a function $f(x)$ is defined as the principal value integral

$$
\mathscr{H}\{f\}(y)=\frac{1}{\pi} \mathrm{PV} \int_{-\infty}^{\infty} \frac{f(x)}{x-y} d x .
$$

We consider primarily the case $f(x) \in L_{2}(-\infty, \infty)$, the set of square integrable functions defined on the real line. For this class of functions the integral (1) exists for almost all $y$, and it defines a function in the same class [7, p. 311]. As for applications, the Hilbert transform plays an important role in optics [4], signal processing [11], and waves in stratified fluids [3, 17].

A list of Hilbert transforms is presented in Table 1 (next page)-it provides the test problems for later sections. The special functions in the table are defined below. Table 1 supplements the one given in Erdélyi et al. [10, Vol. 2] to a modest degree-only the first and last entries of our table can be found in theirs.

In practice the transform can seldom be found in tables such as these. And even when this occurs the result often involves special functions that may pose their own individual challenges to computation; cf. Examples 5-7 in Table 1. Moreover, in many applications $f(x)$ is available only at discrete points $x_{j}$. For these purposes a direct numerical evaluation of (1) is required. The following methods have been proposed:

- Kress and Martensen applied the rectangle rule to (1) to derive

$$
\mathscr{H}\{f\}(y) \approx \frac{2}{\pi} \sum_{n=-\infty}^{\infty} \frac{f(y+(2 n+1) h)}{2 n+1},
$$

where $h$ is the step size [16].

Received by the editor April 16, 1992 and, in revised form, February 21, 1994.

1991 Mathematics Subject Classification. Primary 65R10, 65D30, 44A15.

Key words and phrases. Hilbert transform, orthogonal rational eigenfunctions, Fast Fourier Transform. 
TABLE 1. Hilbert transforms

\begin{tabular}{||c|c|c||}
\hline Ex. & $f(x)$ & $\mathscr{L}\{f\}(y)$ \\
\hline 1 & $\frac{1}{1+x^{2}}$ & $-\frac{y}{1+y^{2}}$ \\
2 & $\frac{1}{1+x^{4}}$ & $-\frac{y\left(1+y^{2}\right)}{\sqrt{2}\left(1+y^{4}\right)}$ \\
3 & $\frac{\sin (x)}{1+x^{2}}$ & $\frac{\cos (y)-e^{-1}}{1+y^{2}}$ \\
4 & $\frac{\sin (x)}{1+x^{4}}$ & $\frac{\cos (y)-e^{-\frac{1}{\sqrt{2}} \cos \left(\frac{1}{\sqrt{2}}\right)-e^{-\frac{1}{\sqrt{2}}} \sin \left(\frac{1}{\sqrt{2}}\right) y^{2}}}{1+y^{4}}$ \\
5 & $e^{-x^{2}}$ & $-\frac{2}{\sqrt{\pi}} D(y)$ \\
6 & $\operatorname{sech}(x)$ & $\tanh (y)+\frac{i}{\pi}\left[\psi\left(\frac{1}{4}+\frac{i y}{2 \pi}\right)-\psi\left(\frac{1}{4}-\frac{i y}{2 \pi}\right)\right]$ \\
7 & $e^{-|x|}$ & $-\frac{1}{\pi} \operatorname{sgn}(y)\left[e^{|y|} E_{1}(|y|)+e^{-|y|} E i(|y|)\right]$ \\
\hline
\end{tabular}

- Stenger derived essentially the same formula, by using expansions in Whittaker's cardinal (or sinc) basis functions [20].

- Henrici proposed a method based on the following well-known property [11, p. 203]. If $f(x) \in L_{2}(-\infty, \infty)$, then

$$
\mathscr{H}\{f\}(y)=\mathscr{F}^{-1}\{i \operatorname{sgn}(k) \mathscr{F}\{f\}(k)\},
$$

almost everywhere, where $\mathscr{F}$ represents the Fourier transform

$$
\mathscr{F}\{f\}(k)=\widehat{f}(k)=\int_{-\infty}^{\infty} f(x) e^{-i k x} d x,
$$

and $\mathscr{F}^{-1}$ its inverse

$$
\mathscr{F}^{-1}\{\widehat{f}\}(x)=f(x)=\frac{1}{2 \pi} \int_{-\infty}^{\infty} \widehat{f}(k) e^{i k x} d k .
$$

These Fourier integrals are approximated by discrete Fourier series on a truncated domain $x \in[-L, L]$. Equidistant gridpoints are used, and the Fast Fourier Transform (FFT) may be used for a rapid summation of the series.

In this paper we introduce what we believe to be a new method for the computation of the Hilbert transform on the real line. It is based on a series expansion in rational eigenfunctions of the Hilbert transform operator in $L_{2}(-\infty, \infty)$. The method is closely related to two other methods for the computation of integral transforms: Weber's method for the computation of Fourier transforms [21], and Weeks' method for the numerical inversion of the Laplace transform [22].

An overview of the paper is as follows: In $\S 2$ we recall that the functions

$$
\rho_{n}(x)=\frac{(1+i x)^{n}}{(1-i x)^{n+1}}, \quad n=0, \pm 1, \pm 2, \ldots,
$$

form a basis for $L_{2}(-\infty, \infty)$. We then show that these functions are eigenfunctions of the Hilbert transform operator in $L_{2}(-\infty, \infty)$. Section 3 is devoted to 
some approximation properties of series expansions involving these eigenfunctions. In particular, we estimate the decay rate of the expansion coefficients for certain classes of functions. In $\S 4$ we introduce the algorithm. It is a collocation method based on a truncated eigenfunction expansion. An attractive property is that the FFT can be used for an efficient implementation. An error analysis for the algorithm is given in $\S 5$. We do not strive for full generality here: we focus merely on predicting the convergence rates for the test problems in Table 1 . In $\S 6$ we compare the methods listed above with the method introduced here. The new method turns out to be more accurate for several test problems. We conclude in $\S 7$ with a synopsis of two applications: the computation of the complex error function, and the solution of a nonlinear wave equation.

A few comments on Table 1 are in order. Entries $1-4$ can be verified straightforwardly using contour integration. The calculations of entries 5 and 7 are given in [18, Ch. 12]. Entry 6 was obtained via a direct computation of (3). The appropriate Fourier transforms may be found on pp. 30 and 88 of [10, Vol. 1].

The special functions in Table 1 are defined as follows: $D(x)$ is Dawson's integral

$$
D(x)=e^{-x^{2}} \int_{0}^{x} e^{t^{2}} d t
$$

and the exponential integrals are defined by

$$
E_{1}(x)=\int_{x}^{\infty} e^{-t} t^{-1} d t, \quad \operatorname{Ei}(x)=-\mathrm{PV} \int_{-x}^{\infty} e^{-t} t^{-1} d t .
$$

The psi (digamma) function $\psi(z)$ is defined in terms of the gamma function as

$$
\psi(z)=\Gamma^{\prime}(z) / \Gamma(z) .
$$

It satisfies $\overline{\psi(z)}=\psi(\bar{z})$, so the transform for $\operatorname{sech}(x)$ listed in Table 1 is indeed a real function.

\section{RATIONAL EIGENFUNCTIONS}

It is well known that the functions

$$
\rho_{n}(x)=\frac{(1+i x)^{n}}{(1-i x)^{n+1}}, \quad n=0, \pm 1, \pm 2, \ldots,
$$

form a complete and orthogonal basis set for $L_{2}(-\infty, \infty)$; see [12, p. 63]. One way to see this is to make the change of variable

$$
e^{i \theta}=\frac{1+i x}{1-i x}, \quad \text { i.e., } \quad x=\tan \frac{1}{2} \theta
$$

which maps the entire real line $x \in[-\infty, \infty]$ to $\theta \in[-\pi, \pi]$. Expansions in terms of $\rho_{n}(x)$ now turn into Fourier series:

$$
f(x)=\sum_{n=-\infty}^{\infty} a_{n} \rho_{n}(x) \Longrightarrow f(x)(1-i x)=\sum_{n=-\infty}^{\infty} a_{n} e^{i n \theta}
$$




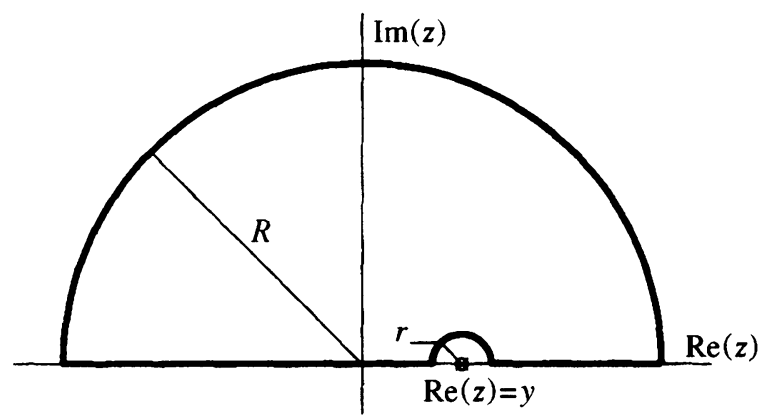

FIGURE 1. Contour of integration used in the proof of Theorem 1

The orthogonality properties of Fourier series may be used to deduce that

$$
\int_{-\infty}^{\infty} \rho_{n}(x) \overline{\rho_{m}(x)} d x=\pi \delta_{n, m},
$$

where the overline denotes the complex conjugate, and $\delta_{n, m}$ is the Kroneckerdelta. This leads to the integral formula for the expansion coefficients

$$
a_{n}=\frac{1}{\pi} \int_{-\infty}^{\infty} f(x) \overline{\rho_{n}(x)} d x .
$$

As for history, the functions $\rho_{n}(x)$ could be traced back to Wiener's book $[26$, p. 35]. Further theoretical properties of these functions are discussed in [6], [12, §2.6.4], and [13, Ch. 19]. Numerical applications are considered in $[8,15,21,23,24,25]$.

What is perhaps not so well known is that the functions $\rho_{n}(x)$ also form a complete set of eigenfunctions of the Hilbert transform operator (1) in $L_{2}(-\infty, \infty)$, with eigenvalues $i \operatorname{sgn}(n)$. The proof is a straightforward exercise in contour integration.

Theorem 1. For all real $y$ and $n=0, \pm 1, \pm 2, \ldots$

$$
\mathscr{C}\left\{\rho_{n}\right\}(y)=i \operatorname{sgn}(n) \rho_{n}(y) .
$$

(We define $\operatorname{sgn}(0)=1$.)

Proof. By definition

$$
\mathscr{H}\left\{\rho_{n}\right\}(y)=\frac{1}{\pi} \mathrm{PV} \int_{-\infty}^{\infty} \frac{(1+i x)^{n}}{(1-i x)^{n+1}(x-y)} d x .
$$

For $n \geq 0$ and fixed $y$ we evaluate this integral using the contour shown in Fig. 1. The contribution along the big semicircle disappears in the limit $R \rightarrow \infty$, and the contribution along the little one approaches $-i \rho_{n}(y)$ as $r \rightarrow 0$. Since the integrand is analytic in the upper half-plane, Cauchy's Theorem yields

$$
\mathscr{H}\left\{\rho_{n}\right\}(y)=i \rho_{n}(y) .
$$

For $n<0$, two semicircles in the lower half-plane are used to complete the proof. 
From the above theorem we deduce that if $f(x) \in L_{2}(-\infty, \infty)$, then

$$
f(x)=\sum_{n=-\infty}^{\infty} a_{n} \rho_{n}(x) \Longrightarrow \mathscr{H}\{f\}(x)=\sum_{n=-\infty}^{\infty} i \operatorname{sgn}(n) a_{n} \rho_{n}(x) .
$$

Our algorithm is based on truncated versions of these series. Implementation details are deferred to $\S 4$.

\section{APPROXIMATION PROPERTIES}

In applications the rate of decay in the coefficients $a_{n}$ in (6) determines the accuracy of the method. However, giving a complete classification of this decay in terms of the usual smoothness properties of $f(x)$ like differentiability, Lipschitz condition, and so on, is no easy task. We remind the reader that even for ordinary Fourier series such a comprehensive theory does not exist (see [2, Ch. 2], for example). We restrict our discussion therefore to estimating the $a_{n}$ for a few simple classes of functions, aimed at covering the test problems in Table 1.

The reader wishing to avoid wading through the details is directed to Fig. 3. There the $a_{n}$ are plotted, together with the asymptotic estimates derived here, for the functions listed in Table 1. Note that in the figure, and also in the analyses below, we consider only $n \geq 0$. We do not sacrifice any generality in doing so, since $a_{n}=\bar{a}_{-1-n}$ for all $n$, provided $f(x)$ is real-valued.

Fig. 3 reveals that the rate of decay in the $a_{n}$ increases in the following order: $f(x)=\sin (x) /\left(1+x^{2}\right), e^{-|x|}, \sin (x) /\left(1+x^{4}\right), \operatorname{sech}(x), e^{-x^{2}}, 1 /\left(1+x^{4}\right)$, and $1 /\left(1+x^{2}\right)$. The accuracy of a numerical method based on (6) is therefore expected to increase in the same order. We confirm this numerically in $\S 5$.

If $f(x)$ is sufficiently regular, methods from analytic function theory may be used for estimating the $a_{n}$, as was pointed out in $[6,21]$. In particular, consider the bilinear map

$$
w=\frac{1+i z}{1-i z}, \quad z=i \frac{1-w}{1+w},
$$

related to (4), with $z=x+i y$. It maps the upper half-plane in the $z$-variable to the unit disc in the $w$-variable. The first expansion in (6) may thus be viewed as a Laurent series:

$$
f(z)=\sum_{n=-\infty}^{\infty} a_{n} \rho_{n}(z) \Longrightarrow g(w)=\sum_{n=-\infty}^{\infty} a_{n} w^{n},
$$

where

$$
g(w)=\frac{2}{1+w} f\left(i \frac{1-w}{1+w}\right) .
$$

Let $R>1$, and consider the regions

$$
\Gamma_{R}: z=i \frac{1-r e^{i \theta}}{1+r e^{i \theta}}, \quad \Lambda_{R}: w=r e^{i \theta},
$$

both defined for $R^{-1}<r<R,-\pi \leq \theta \leq \pi$. These regions are represented by the shaded areas in Fig. 2 (next page); the tick marks in the figure indicate the values $z= \pm i$ and the dot-dash curve represents the unit circle $|w|=1$. 

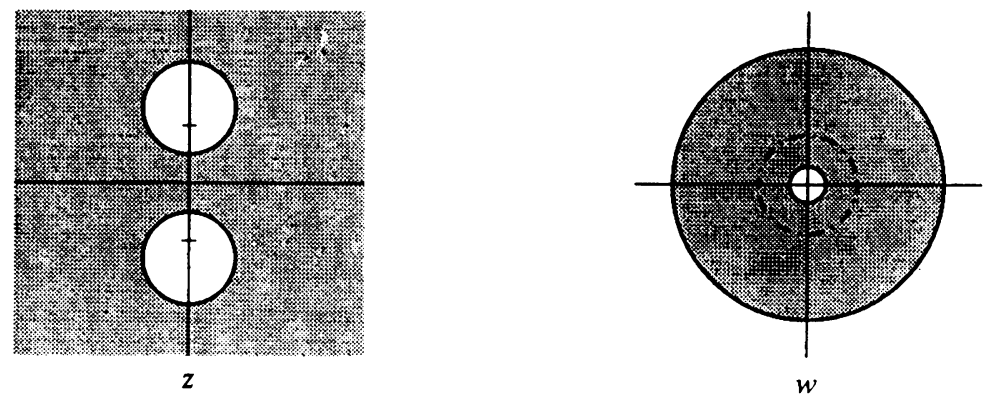

FIgure 2. The regions $\Gamma_{R}$ (left) and $\Lambda_{R}$ (right) defined in (8)

(Similar figures can be found in $[5,6]$.) The domain $\Gamma_{R}$ is mapped conformally to the annulus $\Lambda_{R}$ under the bilinear transformation (7). Here, $\Gamma_{R}$ consists of the exterior of two circles centered at $z= \pm i\left(R^{2}+1\right) /\left(R^{2}-1\right)$, respectively, both with radius $2 R /\left(R^{2}-1\right)$.

If $f(z)$ is analytic in $\Gamma_{R}$, then $g(w)$ is analytic in $\Lambda_{R}$, and the Laurent series converges. Therefore, by Cauchy's estimate [11, Ch. 13]

$$
\left|a_{n}\right| \leq C(r) r^{-n}, \quad C(r) \equiv \max _{|w|=r}|g(w)|,
$$

valid for each $r$ such that $R^{-1}<r<R$. This establishes at least geometric decay in the $a_{n}$. The bigger $R$, the quicker the $a_{n}$ are expected to decay. This means that if the poles of $f(z)$ are clustered near $z= \pm i$, one can expect rapid convergence of the series (6).

The analytic function theory sketched here applies to both Examples 1 and 2 of Table 1. Example 1 represents the extreme case $R=\infty$. Only two of the coefficients $a_{n}$ are nonzero, since $f(z)=1 /\left(1+z^{2}\right) \Longrightarrow g(w)=\frac{1}{2}\left(w^{-1}+1\right)$. Example 2 allows explicit computation of the $a_{n}$ too. We summarize as follows:

Example 1. $f(x)=\frac{1}{1+x^{2}} \quad \Longrightarrow \quad a_{0}=\frac{1}{2}, a_{-1}=\frac{1}{2}$, and $a_{n}=0$ when $n \neq$ $0,-1$.

Example 2. $f(x)=\frac{1}{1+x^{4}} \quad \Longrightarrow$

$$
a_{2 n}=(-1)^{n} \frac{1}{2}(\sqrt{2}-1)^{2 n}
$$

for all $n \geq 0$, and $a_{2 n-1}=-a_{2 n}, a_{-n}=a_{n-1}$.

Though elegant, the above approach to estimating the $a_{n}$ has limited applicability in practice. First, it requires $f(x)$ to be analytic in a neighborhood of the entire real axis, including at $x= \pm \infty$. Only the first two examples in Table 1 meet this requirement; the others have an essential singularity at $x= \pm \infty$. Second, when transplanted from the real line to the unit disk under the mapping (7), functions such as Examples 3-7 in the table assume complicated forms. For these functions different strategies have to be devised.

To deal with Examples 3 and 4, we consider the more general class of functions

$$
f(x)=e^{i \lambda x} \frac{P(x)}{Q(x)},
$$


where $\lambda$ is real, $P(x)$ and $Q(x)$ are polynomials in $x$ with $\operatorname{deg}(Q)>\operatorname{deg}(P)$, and $Q(x)$ has no real zeros. We need to estimate the coefficients

$$
a_{n}=\frac{1}{\pi} \int_{-\infty}^{\infty} \frac{e^{i \lambda x}}{1+i x} \frac{P(x)}{Q(x)}\left(\frac{1-i x}{1+i x}\right)^{n} d x
$$

One approach is repeated integration by parts, based on the identity

$$
\int \frac{1}{1+x^{2}}\left(\frac{1-i x}{1+i x}\right)^{n} d x=-\frac{1}{2 i n}\left(\frac{1-i x}{1+i x}\right)^{n}+C, \quad n \neq 0 .
$$

For example, one step of integration by parts looks like

$$
\int_{-\infty}^{\infty} e^{i \lambda x} \frac{R(x)}{S(x)}\left(\frac{1-i x}{1+i x}\right)^{n} d x=\frac{1}{2 i n} \int_{-\infty}^{\infty} e^{i \lambda x} \frac{\widetilde{R}(x)}{\widetilde{S}(x)}\left(\frac{1-i x}{1+i x}\right)^{n} d x
$$

where $R(x)=P(x)$ and $S(x)=Q(x)(1+i x)$ in the first step. Each successful application of integration by parts reduces the difference between the degrees of the denominator and numerator by two, i.e.,

$$
\operatorname{deg}(\widetilde{S})-\operatorname{deg}(\widetilde{R})=\operatorname{deg}(S)-\operatorname{deg}(R)-2 .
$$

This can be continued as long as $\operatorname{deg}(S)-\operatorname{deg}(R)>2$. Thus it is deduced that for functions of the form (11) one can expect

$$
a_{n}=O\left(n^{-c}\right),
$$

where $c=\left\lfloor\frac{1}{2}(\operatorname{deg}(Q)-\operatorname{deg}(P))\right\rfloor$. This result may be improved, however, as we now show.

We first record the fact that

$$
\int_{-\infty}^{\infty} \frac{e^{i \lambda x}}{(1+i x)^{l}(1-i x)^{m}} d x=\frac{2 \pi \lambda^{2}}{(l-1) !} 2^{1-m-l} e^{-\lambda} U(1-l, 2-m-l, 2 \lambda),
$$

where $m$ and $l$ are integers such that $l+m>1, \lambda>0$, and $U(a, b, x)$ is the confluent hypergeometric function (see $[19$, p. 325]). We also need the asymptotic formula

$$
\begin{aligned}
U(a, b, x)= & \Gamma\left(\frac{1}{2} b-a+\frac{1}{4}\right) \pi^{-1 / 2} e^{\frac{1}{2} x} x^{\frac{1}{4}-\frac{1}{2} b} \\
& \times \cos \left(\sqrt{2 b x-4 a x}-\frac{1}{2} b \pi+a \pi+\frac{1}{4} \pi\right)\left[1+O\left(\left|\frac{1}{2} b-a\right|^{-\frac{1}{2}}\right)\right],
\end{aligned}
$$

as $a \rightarrow-\infty$, for $b$ bounded, $x$ real (see $[1$, p. 508]).

First consider Example 3 of Table 1. We need to evaluate

$$
a_{n}=\frac{1}{\pi} \int_{-\infty}^{\infty} \frac{\sin (x)}{1+x^{2}} \frac{1}{1+i x}\left(\frac{1-i x}{1+i x}\right)^{n} d x=\frac{1}{\pi} \int_{-\infty}^{\infty} \frac{\sin (x)}{(1+i x)^{n+2}(1-i x)^{1-n}} d x
$$

If we split the numerator as $\sin (x)=\left(e^{i x}-e^{-i x}\right) /(2 i)$ and assume $n \geq 0$ the integral involving the term $e^{-i x}$ vanishes. That leaves the integral involving $e^{i x}$, which is in the form (14), with $\lambda=1, l=n+2$, and $m=1-n$. Together with the fact that $\Gamma(n+3 / 4) / \Gamma(n+2) \sim n^{-5 / 4}$, one obtains: 
Example 3. $f(x)=\frac{\sin (x)}{1+x^{2}} \Longrightarrow$

$$
\begin{aligned}
a_{n} & =\frac{1}{4 i e(n+1) !} U(-1-n,-1,2) \\
& =(-1)^{n+1} i \pi^{-1 / 2}(2 n)^{-5 / 4} \cos \left(2(2 n+1)^{1 / 2}-\pi / 4\right)\left[1+O\left(n^{-1 / 2}\right)\right] .
\end{aligned}
$$

Next, consider $f(x)=\sin (x) /\left(1+x^{4}\right)$, and write the rational part as

$$
\frac{1}{1+x^{4}}=\frac{1}{\left(1+x^{2}\right)^{2}}+\frac{2 x^{2}}{\left(1+x^{4}\right)\left(1+x^{2}\right)^{2}} \text {. }
$$

Multiply with $(1 / \pi) \sin (x)(1-i x)^{n} /(1+i x)^{n+1}$ and integrate over $(-\infty, \infty)$; this gives $a_{n}$ on the left. The first integral on the right can be estimated as in Example 3. Integration by parts establishes that the second integral on the right is $O\left(n^{-3}\right)$. Therefore:

Example 4. $f(x)=\frac{\sin (x)}{1+x^{4}} \Longrightarrow$

$$
\begin{aligned}
a_{n} & =\frac{1}{16 i e(n+2) !} U(-2-n,-3,2)+O\left(n^{-3}\right) \\
& =(-1)^{n+1} i \pi^{-1 / 2}(2 n)^{-9 / 4} \cos \left(2(2 n+1)^{1 / 2}-\pi / 8\right)\left[1+O\left(n^{-1 / 2}\right)\right] .
\end{aligned}
$$

It is relatively straightforward to generalize Examples 3 and 4 to obtain a theorem applicable to all functions of the form (11). Since we shall not use such a general result further on, we omit the particulars. It suffices to say that this improves the earlier estimate (13) marginally to $a_{n}=O\left(n^{-c-1 / 4}\right)$.

For functions infinitely differentiable on the real line, and decaying exponentially fast as $|x| \rightarrow \infty$, the integration by parts approach establishes that $a_{n}=O\left(n^{-l}\right)$, for all integers $l$, as $n \rightarrow \infty$. This is a rapid rate of convergence though not necessarily as fast as the geometric rate of Example 2. The precise rate can be obtained by asymptotic methods in some cases, as the author demonstrated for $f(x)=e^{-x^{2}}$ in [24]. A steepest descent analysis was used to predict that $a_{n}=O\left(e^{-(3 / 2) n^{2 / 3}}\right)$, which represents a rapid decay, but not quite the geometric rate of Example 2. A similar analysis establishes that for $f(x)=\operatorname{sech}(x)$ the convergence is slightly slower, namely $a_{n}=O\left(e^{-2 n^{1 / 2}}\right)$. We omit the calculation. The complete estimates are:

Example 5. $f(x)=e^{-x^{2}} \Longrightarrow$

$$
a_{n} \sim(-1)^{n} 2 e^{2 / 3}(3 \pi)^{-1 / 2} m^{-1 / 3} \cos \left(\left(3^{3 / 2} / 2\right) m^{2 / 3}+5 \pi / 3\right) e^{-(3 / 2) m^{2 / 3}},
$$

where $m \equiv n+1 / 2$.

Example 6. $f(x)=\operatorname{sech}(x) \Longrightarrow$

$$
a_{n} \sim(-1)^{n} 2^{3 / 2} \pi^{-1 / 2} n^{-1 / 4} \cos \left(2 n^{1 / 2}+5 \pi / 8\right) e^{-2 n^{1 / 2}} .
$$

Finally, we consider a function with a discontinuity in the first derivative, namely $f(x)=e^{-|x|}$. The coefficients are

$$
a_{n}=\frac{1}{\pi} \int_{-\infty}^{\infty} \frac{e^{-|x|}}{1+i x}\left(\frac{1-i x}{1+i x}\right)^{n} d x=\frac{2}{\pi} \operatorname{Re}\left[\int_{0}^{\infty} \frac{e^{-|x|}}{1+i x}\left(\frac{1-i x}{1+i x}\right)^{n} d x\right] \text {. }
$$




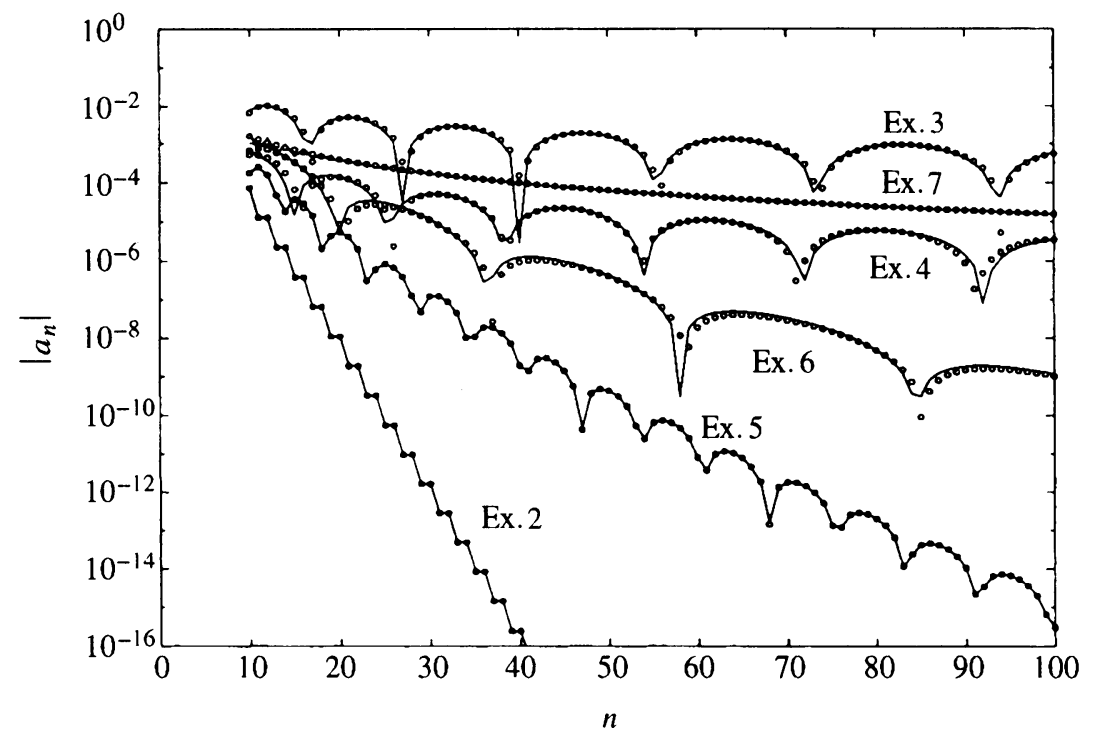

FIGURE 3. Rate of decay in the coefficients $a_{n}, n \geq 10$. The labels refer to the test functions in Table 1 . The continuous lines represent the true values $\left|a_{n}\right|$ and the circles represent the asymptotic approximations given in (15)-(19). Ex. 1 is absent since only two coefficients are nonzero

Integration by parts, based on (12), can be applied to the second integral to conclude that the convergence rate falls somewhere between that of Examples 3 and 4:

Example 7. $f(x)=e^{-|x|} \Longrightarrow$

$$
a_{n}=\frac{1}{2 \pi n^{2}}+O\left(n^{-3}\right) .
$$

As verification of these asymptotic estimates we offer Fig. 3. There is good agreement between the estimated and the actual coefficients, even for relatively small $n$. The exceptions occur near the downward spikes in Examples 3, 4, and 6. For these values of $a_{n}$ the leading terms in the approximations (15), (16), and (18) are no longer dominant, owing to the small cosine factor. Higher-order asymptotics are required here. We computed the "true" coefficients in Fig. 3 using Matlab's FFT (see formula (22) of the next section). A large number of sampling points was used $\left(N=2^{14}\right)$, so that these values can indeed be considered exact, at least on the scale of the figure.

We now introduce the algorithm.

\section{THE ALGORITHM}

The need for computing the Hilbert transform on the real line arises in various ways. For example, $f(x)$ may be known explicitly as a function of $x$, with $\mathscr{H}\{f\}(x)$ to be computed at any $x$. Alternatively, $f(x)$ may be known only at a discrete set $x_{j}$, and $\mathscr{H}\{f\}\left(x_{j}\right)$ needs to be computed, thereby defining a discrete Hilbert transform operator. We first focus on the latter situation, which 
arises for example in the numerical solution of integro-differential equations such as (28) below.

Consider the truncated version of (6), defined by

$$
\mathscr{H}_{N}\{f\}(x)=\sum_{n=-N}^{N-1} i \operatorname{sgn}(n) a_{n} \rho_{n}(x) .
$$

Note that the index ranges over $-N$ to $N-1$. This is the natural truncation, since $\rho_{n}(x)$ and $\rho_{-n-1}(x)$ form a conjugate pair. The coefficients are given by $(5)$, that is

$$
\begin{aligned}
a_{n} & =\frac{1}{\pi} \int_{-\infty}^{\infty} f(x) \overline{\rho_{n}(x)} d x \\
& =\frac{1}{2 \pi} \int_{-\pi}^{\pi}\left(1-i \tan \frac{1}{2} \theta\right) f\left(\tan \frac{1}{2} \theta\right) e^{-i n \theta} d \theta,
\end{aligned}
$$

where we have made the change of variable (4). Assuming that $f(x)$ is available at the collocation points

$$
x_{j}=\tan \frac{1}{2} \theta_{j}, \quad \theta_{j}=\frac{\pi j}{N}, \quad|j|<N,
$$

one may approximate the integral with the trapezoidal rule with spacing $\pi / N$. This yields the approximations $\tilde{a}_{n} \approx a_{n}$, where

$$
\tilde{a}_{n}=\frac{1}{N} \sum_{j=-N+1}^{N-1}\left(1-i \tan \frac{1}{2} \theta_{j}\right) f\left(\tan \frac{1}{2} \theta_{j}\right) e^{-i n \theta_{j}} .
$$

We have set $\left(1-i \tan \frac{1}{2} \theta_{j}\right) f\left(\tan \frac{1}{2} \theta_{j}\right)=0$ when $j= \pm N$, since we are assuming that $f(x)=o(1 / x)$ as $x \rightarrow \pm \infty$.

Once the $\tilde{a}_{n}$ are known, the approximation to (20) may be computed by

$$
\widetilde{\mathscr{X}}_{N}\{f\}\left(x_{j}\right)=\frac{1}{1-i \tan \frac{1}{2} \theta_{j}} \sum_{n=-N}^{N-1} i \operatorname{sgn}(n) \tilde{a}_{n} e^{i n \theta_{j}}, \quad|j|<N .
$$

The linear transformations $f\left(x_{j}\right) \rightarrow \widetilde{a}_{n} \rightarrow \widetilde{\mathscr{H}}_{N}\{f\}\left(x_{j}\right)$ define the discrete Hilbert transform operator. In summary, it is computed as follows: Given the function values $f\left(x_{j}\right)$ at the collocation points (21), compute the coefficients $\tilde{a}_{n}$ according to (22). Then evaluate (23). Both formulas (22) and (23) can be computed with the FFT, rather than separate summations. This reduces the operation count from $O\left(N^{2}\right)$ to $O(N \log N)$.

When $f(x)$ needs to be evaluated at arbitrary $x$, one uses

$$
\widetilde{\mathscr{H}}_{N}\{f\}(x)=\frac{1}{1-i x} \sum_{n=-N}^{N-1} i \operatorname{sgn}(n) \tilde{a}_{n}\left(\frac{1+i x}{1-i x}\right)^{n},
$$

which may be evaluated by Horner's algorithm. 


\section{ERROR ESTIMATES}

The algorithm summarized by (22)-(23) produces the results shown in Fig. 4. We have plotted the error, defined by

$$
\text { error }=\max _{|j|<N}\left|\mathscr{H}\{f\}\left(x_{j}\right)-\widetilde{\mathscr{H}}_{N}\{f\}\left(x_{j}\right)\right|,
$$

as a function of $N$, for the test examples in Table 1. These results, and also those shown in Figs. 5-8 of the next section, were computed with Matlab, with working precision about sixteen digits. The special functions in Table 1 were evaluated with Matlab's Symbolic Toolbox, which is based on the Maple kernel.

It is possible to predict the rate of convergence observed in Fig. 4 in terms of the rate of decay in the coefficients $a_{n}$. To this end we use the following properties: (a) $\left|\rho_{n}(x)\right| \leq 1 / \sqrt{1+x^{2}}$, for all $n$ and $x$. (b) If $f(x)$ is realvalued, then $a_{n}=\bar{a}_{-n-1}$ for all $n \geq 0$, and a similar property applies to the computed coefficients $\tilde{a}_{n}$, for all $n=0, \ldots, N-1$. (c) Assuming that $\sum_{n=-\infty}^{\infty}\left|a_{n}\right|<\infty$, the true coefficients and the computed coefficients are related through [11, p. 19]

$$
\tilde{a}_{n}-a_{n}=\sum_{k=1}^{\infty}\left(a_{n+2 k N}+a_{n-2 k N}\right) .
$$

Theorem 2. Let $\mathscr{H}\{f\}(x)$ and $\widetilde{\mathscr{H}}_{N}\{f\}(x)$ be defined by (6) and (24), and assume that $\sum_{n=-\infty}^{\infty}\left|a_{n}\right|<\infty$. Then, for each real $x$,

$$
\left|\widetilde{\mathscr{H}}_{N}\{f\}(x)-\mathscr{H}\{f\}(x)\right| \leq \frac{4}{\sqrt{1+x^{2}}} \sum_{n=N}^{\infty}\left|a_{n}\right| .
$$

Proof. The error consists of two parts, namely the error associated with using $\tilde{a}_{n}$ instead of $a_{n}$, and the error incurred by truncating the infinite series. These
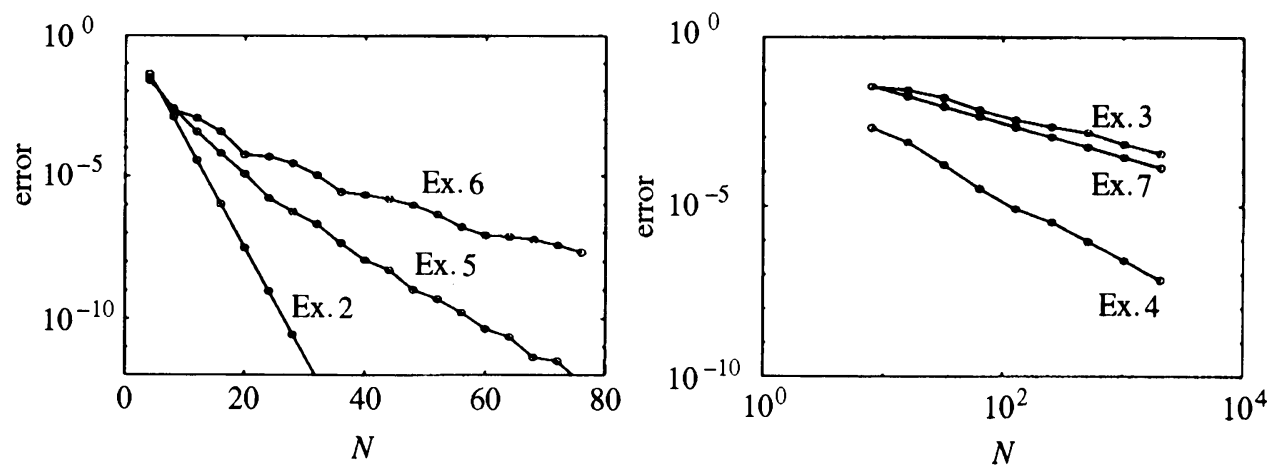

FIGURE 4. The error as defined by (25) as a function of $N$, for the algorithm summarized by (22)-(23). The labels refer to the test examples in Table 1. Notes: (a) Ex. 1 is not represented, since the method is exact with only two terms in the series. (b) The figure on the left is a log-plot, the one on the right is a loglog-plot 
errors may be separated, by writing

$$
\begin{aligned}
& \widetilde{\mathscr{H}}_{N}\{f\}(x)-\mathscr{H}\{f\}(x) \\
& \quad=\sum_{n=-N}^{N-1} i \operatorname{sgn}(n)\left(\widetilde{a}_{n}-a_{n}\right) \rho_{n}(x)+\sum_{n=-\infty}^{-N-1} i a_{n} \rho_{n}(x)-\sum_{n=N}^{\infty} i a_{n} \rho_{n}(x) .
\end{aligned}
$$

By applying the triangle inequality and the properties (a)-(c) listed above, the error bound follows.

In $\S 3$ we estimated the coefficients $a_{n}$ for some simple functions. This enables us to apply the above theorem. For example, suppose the analytic function theory as sketched in (7)-(9) is applicable. That is, we assume there exist positive constants $C$ and $r<1$ such that

$$
\left|a_{n}\right| \leq C r^{n}, \quad n \geq 0 .
$$

By summing the geometric series we establish geometric convergence of our algorithm:

$$
\left|\widetilde{\mathscr{H}}_{N}\{f\}(x)-\mathscr{H}\{f\}(x)\right| \leq \frac{4 C}{\sqrt{1+x^{2}}} \frac{r^{N}}{1-r} .
$$

The estimate (26) applies to Example 2 in Table 1 , with $r=\sqrt{2}-1$; recall (10). The geometric convergence is verified in Fig. 4.

When the coefficients decay algebraically, like

$$
\left|a_{n}\right| \leq C n^{-(p+1)}
$$

for some $p>0$, the analysis is similar, except that we manipulate $p$-series rather than geometric series. This predicts that the error decreases like $O\left(N^{-p}\right)$. This may be confirmed for Example 7 in Fig. 4. The estimate (19) indicates that $p=1$, and a convergence rate of $O\left(N^{-1}\right)$ is evident from the figure. For Examples 3 and 4 we can estimate $p$ to be $1 / 4$ and $5 / 4$ respectively; recall (15) and (16). However, the convergence rates observed in Fig. 4 seem to be better than the predicted $O\left(N^{-1 / 4}\right)$ and $O\left(N^{-5 / 4}\right)$; it appears to be closer to $O\left(N^{-3 / 4}\right)$ and $O\left(N^{-7 / 4}\right)$. This discrepancy can be ascribed to cancellation effects: note the oscillatory nature of the coefficients in (15), (16). Such cancellations are disregarded when the triangle inequality is applied.

Finally, this error analysis suggests that the convergence rates for Examples 5 and 6 are essentially $O\left(e^{-(3 / 2) N^{2 / 3}}\right)$ and $O\left(e^{-2 N^{1 / 2}}\right)$. This is in agreement with the empirical results observed in Fig. 4.

\section{COMPARISONS}

We now compare the methods listed in $\S 1$ with the rational eigenfunction method introduced here. For a fair comparison we note that the rectangle rule (2) and the Fourier-transform method (3) both contain a free parameter that may be adjusted for optimum accuracy. We have the freedom to choose a domain $x \in[-L, L]$ outside of which we set $f(x)=0$. To allow for a similar freedom in the rational expansion, we introduce a modified basis set $\tilde{\rho}_{n}(x)=\rho_{n}(x / L)$. Essentially the only modification to the algorithm given in $\S 4$ is that the collocation points are changed to $x_{j}=L \tan \frac{1}{2} \theta_{j}$. Estimating such 
free parameters is no easy task however; cf. [9, p. 212]. We return to this issue below.

We implement the rectangle rule on an interval $x \in[-L, L]$ using equidistant gridpoints $x_{j}=j h,|j| \leq N$, where $h=L / N$. Formula (2) is therefore computed as

$$
\mathscr{H}_{N}\{f\}\left(x_{j}\right)=\frac{2}{\pi} \sum_{n=-\infty}^{\infty} \frac{f\left(x_{j+2 n+1}\right)}{2 n+1},
$$

where we set $f\left(x_{l}\right)=0$ when $\left|x_{l}\right|>L$. The Fourier-transform method is implemented on the same grid, and the FFT is used to compute the transforms in (3).

In our tests it transpired that the Fourier-transform method (3) often yields very poor results compared to the other two methods, most notably for the test functions $e^{-x^{2}}$ and $\operatorname{sech}(x)$. The problem is that the Hilbert transforms of these two functions are slowly decaying as $|x| \rightarrow \infty$; see Table 1. Such longtailed functions cannot be represented accurately by a discrete Fourier series on a truncated infinite domain. For this reason the Fourier-transform method was eliminated from the tests discussed below.

Comparisons of the remaining two methods are presented in Figs. 5-8. In each case we show the maximum absolute error at the gridpoints (cf. (25)) as a
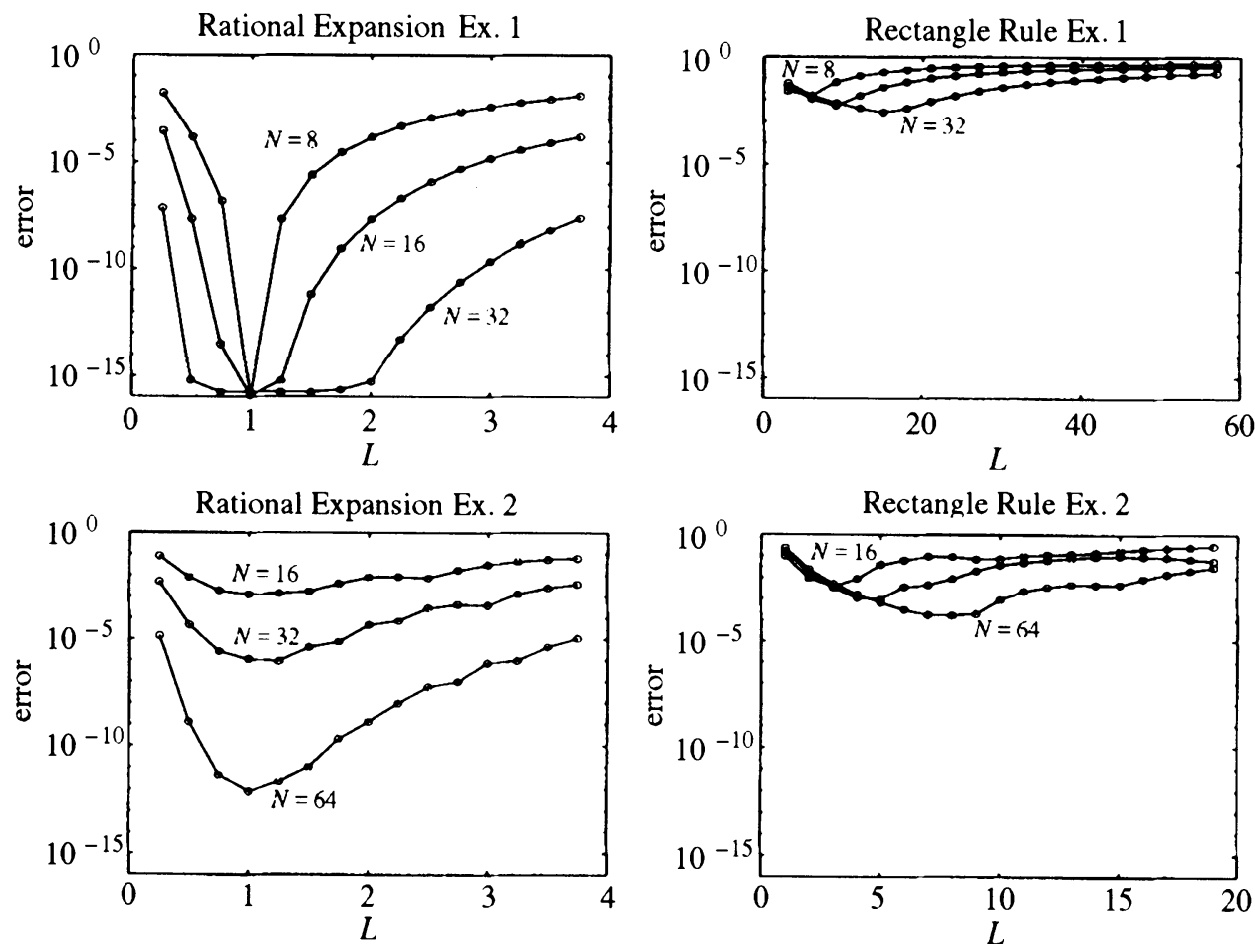

Figure 5. The error as defined by (25) as a function of $L$, for the rational expansion method summarized by (22)-(23) versus the rectangle rule summarized by $(27)$. The test functions are $f(x)=1 /\left(1+x^{2}\right)$ (top) and $f(x)=1 /\left(1+x^{4}\right)$ (bottom) 

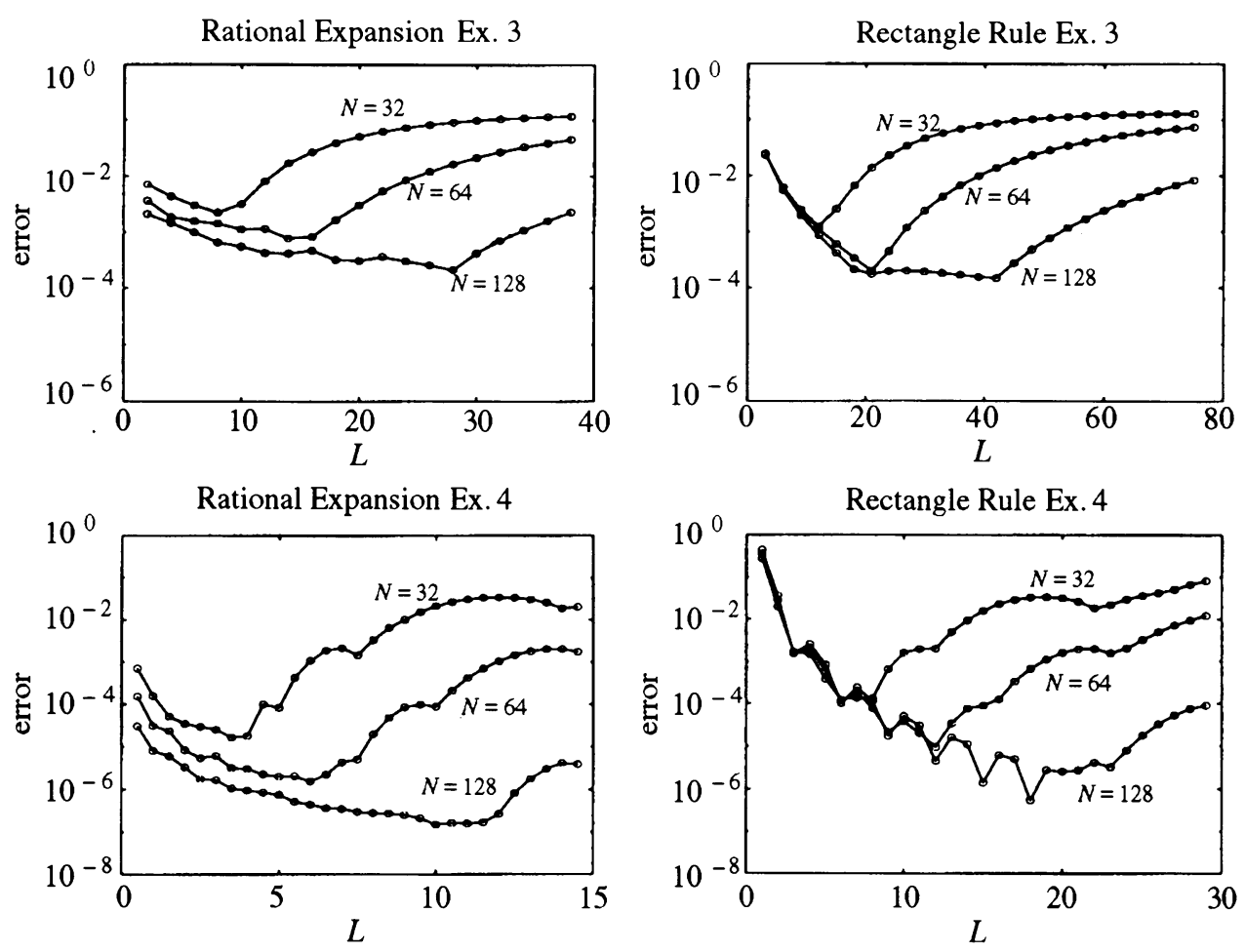

Figure 6. Same as Fig. 5, but the test functions are $f(x)=$ $\sin (x) /\left(1+x^{2}\right)$ (top) and $f(x)=\sin (x) /\left(1+x^{4}\right)$ (bottom)

function of the free parameter $L$, for various $N$. The rational eigenfunction method is superior for Examples 1,2,6, and 7. The rectangle rule is superior for Example 5, and for Examples 3 and 4 there is not much of a difference. For such assessments we assume of course that a near optimal value of $L$ is used.

As for computational efficiency, the rectangle rule and the rational method use respectively $2 N$ and $2 N-1$ function evaluations to compute the transform. However, computing the rectangle rule (27) using straightforward summation requires $O\left(N^{2}\right)$ operations, compared to the $O(N \log N)$ of the eigenfunction method (23). Our Matlab implementation indicated that the eigenfunction method requires fewer operations when $N \geq 128$, provided we restrict $N$ to be a power of two.

To be fair to the rectangle rule we mention two strategies that may improve its efficiency. First, the formula (27) can be viewed as a matrix-vector product involving a Toeplitz matrix. It is possible to perform such a multiplication with the FFT in $O(N \log N)$ operations, provided one embeds the Toeplitz matrix in a circulant matrix of twice the size. Such an approach will only be efficient for $N$ much bigger than the values considered here. Second, it may be possible to apply some form of convergence acceleration to the summation (27). However, this requires prior knowledge of the function. When solving integro-differential equations, for example, the function is an unknown and such information may not be readily available. 

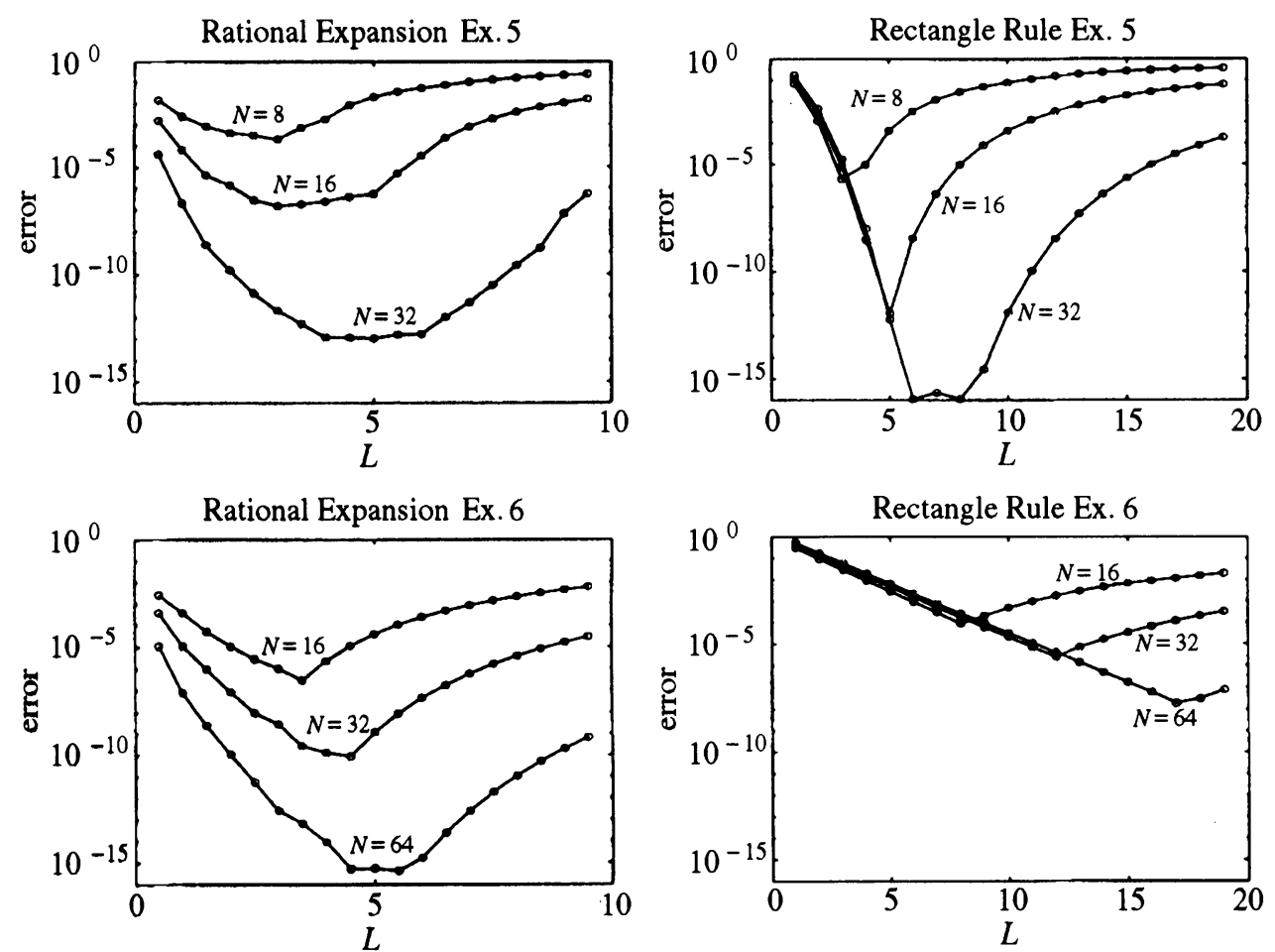

Figure 7. Same as Fig. 5, but the test functions are $f(x)=e^{-x^{2}}$ (top) and $f(x)=\operatorname{sech}(x)$ (bottom)
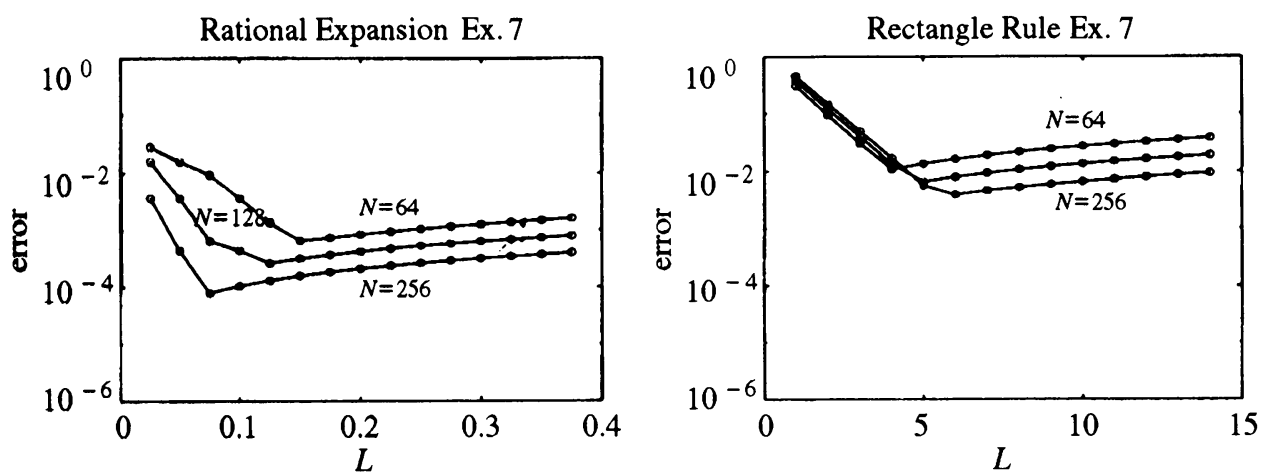

Figure 8. Same as Fig. 5, but the test function is $f(x)=e^{-|x|}$

The rational expansion method has two further advantages. First, it obviates the need for selecting a relationship $L$ vs. $N$. A fixed value of $L$ leads to convergence as $N \rightarrow \infty$ for each of the test Examples 1-7. This is not true for the rectangle rule: keeping $L$ fixed as $N \rightarrow \infty$ does not lead to convergence as is clear from Figs. 5-8. Second, the rational eigenfunction method can be used to compute asymptotic values of $\mathscr{H}\{f\}(x), x \rightarrow \pm \infty$. Consider for example $\mathscr{H}\left\{e^{-x^{2}}\right\}(x)=-(2 / \sqrt{\pi}) D(x)$, and suppose we would like to confirm that $D(x) \sim 1 /(2 x), x \rightarrow \infty$. Define the approximate Dawson function $D_{N}(x)$ 
TABLE 2. Numerical verification of $2 x D(x) \sim 1$ as $x \rightarrow \infty$

\begin{tabular}{||c|c||}
\hline$x$ & $2 x D_{N}(x)-1$ \\
\hline 10 & $5.1(-3)$ \\
100 & $5.0(-5)$ \\
1000 & $5.0(-7)$ \\
\hline
\end{tabular}

through $\widetilde{\mathscr{H}}_{N}\left\{e^{-x^{2}}\right\}(x)=-(2 / \sqrt{\pi}) D_{N}(x)$, and evaluate (24) with Horner's rule. With $N=64, L=1$, this produces the results in Table 2. It is not clear how such large- $x$ calculations can be performed efficiently with the rectangle rule or the Fourier-transform method.

\section{CONCLUSIONS}

In this paper we introduced what we believe to be a new method for the computation of the Hilbert transform on the real line. Convergence rates for some simple classes of functions were established, and these results were verified numerically for the test problems in Table 1.

The method was found to yield higher accuracy than its competitors in several (but not all) test problems considered. In addition, it has several attractive features which sets it apart from its rivals: First, no grid refinement strategy ( $L$ vs. $N$ ) is required. Second, it is useful for asymptotic calculations such as those presented in Table 2 . Third, it can be implemented by the FFT.

We also presented some preliminary results on the rate of decay of the expansion coefficients $a_{n}$ for some test functions $(\S 3)$. These estimates are not only applicable to the calculation of the Hilbert transform. It may also be useful for computing Fourier transforms using Weber's method [21], for the inversion of the Laplace transform with Weeks' method [22], or for solving differential equations using expansions in the basis functions $\rho_{n}(x)[5,8,23]$.

One problem that has not been resolved completely is the selection of the optimal parameter $L$ in our method. As is clear from Figs. 5-8, the optimal $L$ typically depends on $N$, but in general the precise relationship is hard to determine. However, for individual test examples asymptotic analysis may provide the answer. The case $f(x)=e^{-x^{2}}$ was analyzed in detail in [24]. The optimal choice was shown to be $L=2^{-1 / 4} N^{1 / 2}$, which correlates well with the numerical results presented in Fig. 7.

We conclude with some remarks on two applications, namely the complex error function and the Benjamin-Ono equation. Certain refinements of our algorithm lead to a very efficient algorithm for calculating the complex error function $w(z)=e^{-z^{2}} \operatorname{erfc}(-i z)$, which can be represented as a Hilbert transform of $f(x)=e^{-x^{2}}$ extended into the complex plane. See [24, 25] for details.

We also implemented the method on the Benjamin-Ono (BO) equation

$$
u_{t}+u u_{x}+\mathscr{H}\left\{u_{x x}\right\}=0, \quad-\infty<x<\infty,
$$

which, together with a suitable initial condition $u(x, 0)$, describes waves in stratified fluids [3, 17]. This equation is similar to the well-known Kortewegde Vries equation (KdV), which replaces the term $\mathscr{H}\left\{u_{x x}\right\}$ with $u_{x x x}$. Like the $\mathrm{KdV}$ equation the $\mathrm{BO}$ equation admits soliton solutions. These are solitary wave solutions which preserve their shape during nonlinear interaction. However, 
the BO solitons are algebraic, i.e., rational functions in $x$ and $t$ (see [17]). By contrast $\mathrm{KdV}$ solitons decay exponentially as $|x| \rightarrow \infty$.

This rational nature of the $\mathrm{BO}$ solitons makes the present method especially suitable for the $\mathrm{BO}$ equation. The function $u(x, t)$ was expanded in terms of the basis functions $\rho_{n}(x)$ with time-dependent coefficients. The $x$-derivatives were computed by the methods described in $[8,15,23]$, and the Hilbert transform was computed by the method introduced here. This led to a nonlinear system of ordinary differential equations in the $t$ variable, which we integrated with the explicit midpoint method. This approach turned out to be very accurate, at least in the initial stages before the waves move too far from the origin. When this occurs, the increasing grid-spacing (recall $x_{j}=\tan (\pi j / 2 N)$ ) leads to a lack of resolution, and the quality of the approximation deteriorates. Numerical results are presented in [15].

\section{ACKNOWLEDGMENTS}

The author acknowledges Rodney James, whose paper on the Benjamin-Ono equation initiated this research [14]. Enrique Thomann and an anonymous referee both offered many useful suggestions.

\section{BIBLIOGRAPHY}

1. M. Abramowitz and I. Stegun, Handbook of mathematical functions, Dover, New York, 1972.

2. N.K. Bary, A treatise on trigonometric series, Vol. I, Macmillan, New York, 1964.

3. T. B. Benjamin, Internal waves of permanent form in fluids of great depth, J. Fluid. Mech. 25 (1967), 559-592.

4. C. F. Bohren and D. R. Huffman, Absorption and scattering of light by small particles, Wiley-Interscience, New York, 1983.

5. J. Boyd, Spectral methods using rational basis functions on an infinite interval, J. Comput. Phys. 69 (1987), 112-142.

6. - The orthogonal rational functions of Higgins and Christov and algebraically mapped Chebyshev polynomials, J. Approx. Theory 61 (1990), 98-105.

7. P. L. Butzer and R. J. Nessel, Fourier analysis and approximation, Vol. I, Academic Press, New York, 1971.

8. C. I. Christov, A complete orthonormal system of functions in $L^{2}(-\infty, \infty)$ space, SIAM J. Appl. Math. 42 (1982), 1337-1344.

9. P. J. Davis and P. Rabinowitz, Methods of numerical integration, 2nd ed., Academic Press, San Diego, 1984.

10. A. Erdélyi, W. Magnus, F. Oberhettinger, and F. G. Tricomi, Tables of integral transforms, Vols. I and II, McGraw-Hill, New York, 1954.

11. P. Henrici, Applied and computational complex analysis, Vol. III, Wiley-Interscience, New York, 1986.

12. J. R. Higgins, Completeness and basis functions of sets of special functions, Cambridge Univ. Press, Cambridge, 1977.

13. E. Hille, Analytic function theory, Vol. II, Ginn, Boston, 1962.

14. R. James, Pseudospectral methods for the Benjamin-Ono equation, Master's Paper, Oregon State Unversity (unpublished).

15. R. James and J. A. C. Weideman, Pseudospectral methods for the Benjamin-Ono equation, Advances in Computer Methods for Partial Differential Equations-VII (R. Vichnevetsky, D. Knight, and G. Richter, eds.), IMACS, New Brunswick, 1992, pp. 371-377. 
16. R. Kress and E. Martensen, Anwendung der Rechteckregel auf die reelle Hilberttransformation mit unendlichem Intervall, Z. Angew. Math. Mech. 50 (1970), T61-T64.

17. H. Ono, Algebraic solitary waves in stratified fluids, J. Phys. Soc. Japan 39 (1975), 10821091.

18. A. C. Pipkin, A course on integral equations, Springer-Verlag, New York, 1991.

19. A. P. Prudnikov, Y. A. Brychkov, and O. I. Marichev, Integrals and series, Vol. I: Elementary functions, Gordon and Breach, New York, 1986.

20. F. Stenger, Approximations via Whittaker's cardinal function, J. Approx. Theory 17 (1976), 222-240.

21. H. Weber, Numerical computation of the Fourier transform using Laguerre functions and the fast Fourier transform, Numer. Math. 36 (1981), 197-209.

22. W. T. Weeks, Numerical inversion of Laplace transforms using Laguerre functions, J. Assoc. Comput. Mach. 13 (1966), 419-429.

23. J. A. C. Weideman, The eigenvalues of Hermite and rational spectral differentiation matrices, Numer. Math. 61 (1992), 409-432.

24. _ Computation of the complex error function, SIAM J. Numer. Anal. 31 (1994), 14971518.

25. Computing integrals of the complex error function, Mathematics of Computation 1943-1993: A half-century of computational mathematics (W. Gautschi, ed.), Proc. Sympos. Appl. Math., vol. 48, 1994, pp. 403-407.

26. N. Wiener, Extrapolation, interpolation, and smoothing of stationary time series, M.I.T. Press, Cambridge, 1949.

Department of Mathematics, Oregon State University, Corvallis, Oregon 97331-4605

E-mail address: weideman@math.orst.edu 